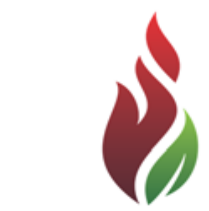

SUSTENERE

Publishing Corporation
SRBADM

Journals Homepage:

www.sustenere.co/journals

\section{PRODUÇÃO CIENTÍFICA SOBRE CULTURA ORGANIZACIONAL: UMA ANÁLISE DAS PUBLICAÇÕES BRASILEIRAS, 2008-2011}

\section{RESUMO}

Este estudo tem por objeto a análise da produção científica brasileira sobre cultura organizacional. Trata-se de levantamento, por meio de técnicas bibliométricas, de artigos publicados sobre cultura organizacional de 2008 a 2011, disponibilizada no Portal Scientific Periodicals Eletronic Library - SPELL. Dos 6568 artigos em língua portuguesa disponíveis no Portal SPELL, 50 são sobre cultura organizacional. Foram encontrados artigos de 26 periódicos, sendo que, 6 deles concentravam $48 \%$ da produção, assim distribuídos: Revista de Administração Pública (10\%), Revista Eletrônica de Ciência Administrativa (10\%), Revista de Administração da UNIMEP (8\%), Revista de Administração Mackenzie (8\%), Revista de Administração da UFSM $(6 \%)$ e a Revista Alcance $(6 \%)$. Entre os temas abordados com maior frequência destacam-se: cultura nacional, mudança organizacional e aprendizagem organizacional. A ampla maioria dos autores possui formação em Administração e estão vinculados às universidades. A pesquisa foi exploratória, com método qualitativo, por meio de análise documental, no primeiro momento e descritiva, com método quantitativo, num segundo momento. A análise considerou número de artigos, autores, temas, metodologia, instrumentos de coleta de dados, populações alvo e área de formação dos autores. Os trabalhos estão voltados tanto para subsidiar intervenções como para impulsionar o desenvolvimento teórico da área.

PALAVRAS-CHAVES: Cultura Organizacional; Produção Científica; SPELL.

\section{SCIENTIFIC LITERATURE ON ORGANIZATIONAL CULTURE: AN ANALYSIS OF BRAZILIAN PUBLICATIONS, 2008 - 2011}

\begin{abstract}
This study has as objective the analysis of Brazilian scientific production on organizational culture. It is about raising through bibliometric techniques of published articles on organizational culture from 2008 to 2011, available on the Portal Scientific Electronic Library Periodicals - SPELL. Of the 6568 articles in Portuguese available on Portal SPELL, 50 are about organizational culture. Articles of 26 journals were found, 6 of them concentrated $48 \%$ of production, as follows: Electronic Magazine of Public Administration (10\%), Electronic Journal of Administrative Science $(10 \%)$, Journal of Administration of UNIMEP (8\%), Mackenzie Administration Magazine $(8 \%)$, Management Magazine UFSM (6\%) and Range Magazine (6\%). Among the topics discussed most frequently are: national culture, organizational change and organizational learning. The vast majority of authors have training in management and are linked to universities. The research was exploratory, with qualitative method through document analysis, in the first moment and descriptive with quantitative method, in a second moment. The analysis considered the number of articles, authors, themes, methodology, data collection instruments, target populations and area of its authors. The work is directed both to support interventions for boosting the theoretical development of the area.
\end{abstract}

KEYWORDS: Organizational Culture; Scientific Production; SPELL.
Revista Brasileira de

Administração Científica,

Aquidabã, v.5, n.3, Jul, Ago, Set,

Out, Nov, Dez 2014.

ISSN 2179-684X

SECTION: Articles

TOPIC: Teoria Geral da

Administração

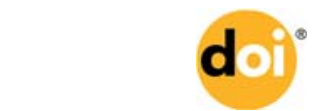

DOI: 10.6008/SPC2179-684X.2014.003.0001

Ítalo de Paula Casemiro

Universidade Federal de Rondônia, Brasil http://lattes.cnpq.br/0400215407886003

itcasemiro@hotmail.com

Angelina Maria de Oliveira Licorio Universidade Federal de Rondônia, Brasil http://lattes.cnpq.br/5999105777814994 clicorio@gmail.com

Osmar Siena

Universidade Federal de Rondônia, Brasil http://lattes.cnpq.br/5424632182909652 osmar siena@uol.com.br

Received: 01/05/2014

Approved: 15/11/2014

Reviewed anonymously in the process of blind peer.

Referencing this:

CASEMIRO, I. P.; LICORIO, A. M. O.; SIENA, O.. Produção científica sobre cultura organizacional: uma análise das publicações brasileiras, 2008-2011. Revista Brasileira de Administração Científica, Aquidabã, v.5, n.3, p.7-17, 2014. DOl: http://dx.doi.org/10.6008/SPC2179 684X.2014.003.0001 


\section{INTRODUÇÃO}

A partir de um breve levantamento das publicações disponíveis no Portal SPELL (Scientific Periodicals Eletronic Library), percebe-se que existe um número baixo de produção abordando a temática da cultura organizacional, quando comparado ao número de artigos disponíveis na referida base. O contexto organizacional contemporâneo requer, a cada dia, a adequação das empresas às mudanças decorrentes de transformações no mercado, políticas governamentais, exigências dos clientes, colaboradores e outras entidades sociais, o que exige constantemente revisões no modus operandi das organizações em todo o mundo. Dentro deste novo cenário, o estudo da cultura organizacional torna-se um dos aspectos mais importantes da organização para o direcionamento das suas atividades, pois orienta as decisões de gestão em todos os níveis da estrutura organizacional (BELTRÃO \& CARDOSO, 2009).

Analisando a produção científica publicada em periódicos nacionais, poder-se-á observar algumas tendências como predominância de pesquisas qualitativas, as investigações quantitativas são menos frequentes, assim como as pesquisas que combinam os dois métodos: qualitativo e quantitativo. Outra tendência é a diversificação de assuntos atrelados à cultura organizacional, destacando-se um número maior de estudos sobre cultura nacional, mudança organizacional e aprendizagem organizacional.

Dado o papel central da investigação científica e a relevância do tema proposto, o propósito e objetivo geral deste estudo consistem em levantar informações sobre a produção científica contemporânea em cultura organizacional, publicadas especificamente em periódicos nacionais voltados a área da Administração de Empresas no período de 2008 a 2011. Para a realização do estudo foram utilizadas as publicações disponíveis no Portal SPELL (Scientific Periodicals Eletronic Library). Nesse sentido o presente estudo identificou por meio do uso de técnicas bibliométricas: o volume de publicações sobre o tema, os autores dos artigos identificando os mais produtivos; os temas adjacentes à cultura organizacional; os periódicos com maior produtividade; os métodos de pesquisa utilizados; os instrumentos de coleta de dados adotados; as populações-alvo escolhidas para o estudo; e por fim, foram examinadas as áreas de formação do primeiro autor por meio de consulta ao Currículo Lattes.

Justifica-se a escolha do Portal SPELL por ser um sistema de indexação, pesquisa e disponibilização gratuita da produção científica e que busca promover o acesso, organização, disseminação e análise da produção científica de distintas áreas do conhecimento. Além disso, o Portal SPELL iniciou em 2012 e concentra inicialmente, a produção científica das áreas de Administração, Contabilidade e Turismo, publicadas a partir de 2008, por este motivo nosso estudo compreenderá a produção sobre cultura organizacional de 2008 a 2011. Assim este estudo busca contribuir para a pesquisa sobre cultura organizacional, analisando uma parte relevante da produção científica brasileira. 
O artigo foi estruturado em quatro partes. Inicialmente apresentou-se uma visão geral sobre as técnicas bibliométricas e seu uso no estudo da produção científica de uma área, para em seguida, desenvolver o tema central do estudo que é a cultura organizacional. Posteriormente delineou a metodologia empregada no estudo e em seguida descreveu-se o resultado obtidos nesta pesquisa. Por fim, foram apresentadas as conclusões e as recomendações do trabalho realizado.

\section{METODOLOGIA}

Os dados do presente estudo são constituídos de artigos publicados em periódicos nacionais entre 2008 a 2011, disponíveis no Portal SPELL (Scientific Periodicals Eletronic Library) em dezembro de 2012. A inclusão das publicações sobre cultura organizacional foi definida a partir da análise dos títulos, resumos e palavras-chave dos artigos publicados em periódicos nacionais. Para a análise foram utilizados apenas artigos. Cabe salientar que o Portal SPELL também disponibiliza, além de artigos, resenhas, resumos entre outras publicações.

Este estudo pode ser caracterizado, inicialmente, como exploratório, com método qualitativo. Esta pesquisa exploratória qualitativa é do tipo documental, visto que o material objeto de estudo são artigos publicados revistas científicas, um tipo de publicação periódica. Em um segundo momento, a pesquisa assume caráter descritivo, com método quantitativo.

Como o objetivo geral do estudo foi analisar a produção sobre cultura organizacional no Brasil, na etapa exploratória e qualitativa da pesquisa realizou-se um levantamento dos artigos científicos publicados em periódicos nacionais e disponibilizados no Portal SPELL. Para tanto, optou-se por analisar os artigos produzidos entre 2008 e 2011, visto que este período é onde deu início a concentração de publicações no referido portal.

Num primeiro momento da pesquisa, buscou-se qualificar todos os artigos científicos sobre cultura organizacional, disponibilizados no portal. Assim, procedeu-se a filtragem, por busca de artigos sobre o tema em questão, por ano e idioma. Após esta abordagem os artigos foram separados para análise. Nestas condições, encontrou-se um total de 50 artigos científicos. Estes 50 artigos passaram a integrar a segunda etapa da pesquisa, sendo esta descritiva quantitativa. Nesta etapa, a finalidade inicial foi identificar, nos artigos em estudo, as seguintes variáveis: o volume de publicações sobre o tema, os autores dos artigos, quais eram os mais produtivos; os temas adjacentes à cultura organizacional tratado; os periódicos com maior produtividade sobre o tema; os métodos de pesquisa utilizados; os instrumentos de coleta de dados adotados; as populações-alvo escolhidas para o estudo; e por fim, foram examinadas as áreas de formação do primeiro autor por meio de consulta ao Currículo Lattes. Em seguida, foi realizada a quantificação dos resultados, com a contagem simples de frequência. Ao concluir esta etapa, em cada um dos artigos avaliados, os dados foram analisados por meio de estatísticas descritivas, utilizando-se 
como apoio o software Microsoft Excel. Os resultados foram reunidos em tabelas e descritos, sucintamente.

Quanto aos métodos de pesquisa utilizados nos estudos publicados observa-se que os métodos do tipo qualitativo e exploratório são a preferência da maioria dos autores, conforme tabela 1. Este fato se explica porque na maioria dos artigos o objetivo dos autores era obter maior familiaridade com o problema para torná-lo explícito. O levantamento demonstrou que os estudos qualitativos representam ampla maioria com $82 \%$ de participação, enquanto os estudos quantitativos representam apenas $10 \%$. Ressalta-se que $8 \%$ dos artigos apresentam os dois tipos de estudo: qualitativos e quantitativos combinados. Destaca-se que houve um grande emprego de estudos de caso na produção dos artigos, estando presente em $40 \%$ dos artigos.

Tabela 1: Distribuição de Metodologia Empregada nos Artigos.

\begin{tabular}{|l|l|l|}
\hline MÉTODOS DE PESQUISA & $\mathbf{f}($ TOTAL) & f (\%) \\
\hline Qualitativos & 41 & $82 \%$ \\
\hline Quantitativos & 5 & $10 \%$ \\
\hline Misto & 4 & $8 \%$ \\
\hline Descritivos & 21 & $42 \%$ \\
\hline Exploratórios & 22 & $44 \%$ \\
\hline Estudos de Caso & 20 & $40 \%$ \\
\hline Etnográficos & 5 & $10 \%$ \\
\hline Outros & 4 & $8 \%$ \\
\hline
\end{tabular}

\section{Instrumentos de Coleta de Dados}

Analisando os instrumentos de coleta de dados adotados pelos autores percebe-se que a pesquisa documental, as entrevistas semiestruturadas e os questionários, são os instrumentos mais utilizados pelos pesquisadores, conforme demonstrado na tabela 2. Como a maioria dos artigos são de caráter qualitativo e exploratório, há uma preferência por entrevistas, questionários e estudos de exemplos como instrumento de coleta de dados mais comum nesta modalidade de pesquisa.

Tabela 2: Distribuição de Instrumentos de Coletas de Dados Utilizados nos Artigos.

\begin{tabular}{|l|l|l|}
\hline INSTRUMENTOS & $\mathbf{f}($ TOTAL) & $\mathbf{f ( \% )}$ \\
\hline Entrevista Semiestruturada & 23 & $46 \%$ \\
\hline Entrevista Pessoal com Perguntas Fechadas & 7 & $14 \%$ \\
\hline Observação Participante & 7 & $14 \%$ \\
\hline Observação Não-Participante & 9 & $18 \%$ \\
\hline Questionários & 17 & $34 \%$ \\
\hline Pesquisa Documental & 31 & $62 \%$ \\
\hline Outros & 5 & $10 \%$ \\
\hline
\end{tabular}

\section{População Alvo}

Por este critério identificou-se que o tipo de estudo interfere diretamente na população alvo, por exemplo, nos estudos do tipo etnográfico, as populações alvo foram ao todo, os funcionários das organizações, pois este é um tipo de estudo que se caracteriza pelo envolvimento entre o pesquisador e a população alvo do seu estudo. Conforme Laplantine (1995, 
p.150): "O etnógrafo é aquele que deve ser capaz de viver nele mesmo a tendência principal da cultura que estuda. Se, por exemplo, a sociedade tem preocupações religiosas, ele próprio deve rezar com seus hóspedes". Na tabela 3 demonstra-se a distribuição da população alvo.

Tabela 3: Distribuição da População Alvo das Pesquisas.

\begin{tabular}{|l|l|l|}
\hline POPULAÇÕES & $\mathbf{f}($ TOTAL) & $\mathbf{f}(\%)$ \\
\hline Empresas & 33 & $66 \%$ \\
\hline Funcionários & 15 & $30 \%$ \\
\hline Outros & 10 & $20 \%$ \\
\hline
\end{tabular}

\section{Distribuição de Artigos por Periódicos: Formação dos Autores}

Quanto à área de formação superior inicial (primeira titulação em nível superior) do primeiro autor, obtida por meio de consulta ao Currículo Lattes, observou-se que a maioria dos autores dos artigos analisados são bacharéis em Administração, representando $75 \%$ do total, conforme vemos no Gráfico 1. Constatou-se ainda, que grande parte deles são professores ou estão vinculados a instituições de ensino superior. Outro dado importante é que a maioria dos autores é proveniente de instituições públicas. Verificou-se também, que a produção nacional sobre cultura organizacional apresenta uma concentração em instituições das regiões Sudeste, Sul e Nordeste do país, respectivamente.

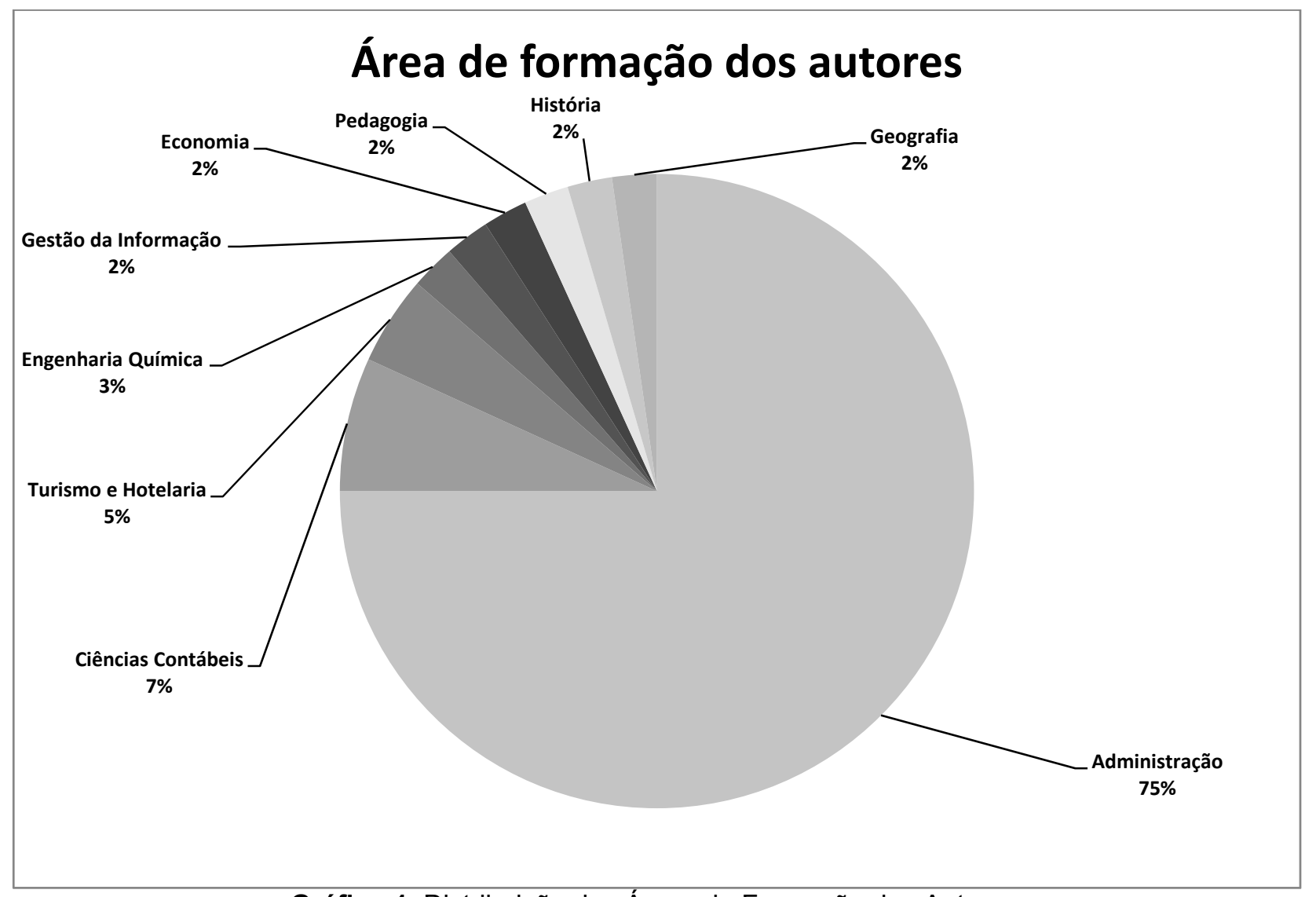

Gráfico 1: Distribuição das Áreas de Formação dos Autores. 


\section{DISCUSSÃO TEÓRICA}

A cultura organizacional constitui elemento importante no contexto organizacional, o que leva diversos autores a estudarem esta temática de modo contínuo e sistemático. Um dos caminhos a ser adotado na busca de se compreender a temática é o da bibliometria que segundo Araujo (2006, p.12) refere-se a uma "técnica quantitativa e estatística de medição dos índices de produção e disseminação do conhecimento científico".

\section{A Bibliometria na Análise da Produção Científica}

Destaca-se como principais leis da bibliometria, a lei de Lotka voltada para o estudo da produtividade científica dos autores, a lei de Bradford voltada para a análise da produtividade de periódicos, e a Lei de Zipf que tem como foco a identificação da frequência de palavras no estudo. Pelo uso das técnicas bibliométricas torna-se possível a medição da produtividade e da dispersão do conhecimento da produção científica e a identificação do modelo de distribuição e frequência de palavras em um texto. A Lei de Lotka, também conhecida como Lei do Quadrado Inverso foi formulada em 1926 referindo-se a uma larga produção científica originada de um pequeno número de autores. (ARAUJO, 2006). A Lei de Zipf, também conhecida como Lei do Menor Esforço preocupa-se em aferir a frequência com que determinadas palavras aparecem na literatura estudada. Bradford fez suas primeiras observações sobre a dispersão de artigos em 1934, contudo, somente em 1948 foi reconhecido com o status de lei. Assim dispõe o enunciado da lei:

[...] se os periódicos forem ordenados em ordem de produtividade decrescente de artigos sobre um determinado assunto, poderão ser distribuídos num núcleo de periódicos mais particularmente devotados a esse assunto e em diversos grupos ou zonas contendo o mesmo número de artigos que o núcleo, sempre que o número de periódicos e das zonas sucessivas for igual a 1:n:n2. (PINHEIRO, 1983 citado por FERREIRA, 2010, p.4).

Para Bernardino e Cavalcante, a "Bibliometria é o estudo dos aspectos quantitativos da produção, disseminação e uso da informação registrada; desenvolve padrões e modelos matemáticos para medir esses processos, usando seus resultados para elaborar previsões e apoiar tomadas de decisão" (2011, p.251). Desta forma é possível, pela bibliometria, delinear um cenário seguro da produção científica sobre a Cultura Organizacional no país, capaz de nortear pesquisadores e editoras, entre outros, sobre temas a merecerem novos estudos.

O uso de técnicas bibliométricas, segundo Figueiredo (1993) permite a análise do desenvolvimento e da distribuição da produção científica, considerando, ao mesmo tempo, a estrutura social dos que realizam essa produção e a interação existente entre os que produzem e os que consomem este conteúdo. O estudo bibliométrico, objetiva medir e quantificar os resultados para minimizar as possíveis distorções quando da análise e interpretação dos dados (GNECCO JÚNIOR et al., 2010). Por este estudo têm-se o uso de técnicas estatísticas e matemáticas para objetivamente analisar a produção cientifica. 


\section{A Cultura Organizacional}

O tema cultura organizacional tem sido vastamente abordado na literatura. Existem diversos correntes e estudiosos que abordam o tema de maneiras distintas, em alguns casos até conflitantes. Mas, de forma geral, existe um consenso de que as crenças, os valores e as normas de comportamento originam a cultura organizacional, de forma a determinar os referenciais que orientam os processos de gestão e as atividades administrativas. Além do que pode ser uma barreira eficaz à imitação, pois cada empresa tem uma cultura singular e intangível, de difícil reprodução pela concorrência. Desde a década de 80 , quando surgiram os primeiros estudos sobre cultura organizacional, foi the atribuída a capacidade de solucionar todos os problemas organizacionais. Inúmeros estudos vêm sendo realizados como forma de compreender os aspectos mais importantes deste construto, que se revela cada dia mais interessante, do ponto de vista da pesquisa científica (SILVA \& FADUL, 2010).

Para Robbins (1990, p.46) "a cultura é definida como as atitudes, crenças e valores de uma sociedade. Estes incluem as diferenças psicológicas individuais, tais como traços de personalidade e, evidentemente, os diversos sistemas sociais, políticos e educacionais da sociedade". Por sua vez, Morgan (1996) ao falar de cultura, caracteriza-a como o padrão de desenvolvimento refletido nos sistemas sociais de conhecimento, ideologia, valores, leis e rituais quotidianos. Nesta perspectiva, o autor relata que o fenômeno conhecido como cultura organizacional estabelece que as organizações como pequenas sociedades que têm os seus próprios padrões distintos de cultura e subcultura (MACIEL et al., 2011). Outra definição amplamente utilizada para definir a cultura organizacional, afirma que ela:

[...] é formada pelo conjunto de pressupostos básicos que um grupo inventou, descobriu ou desenvolveu, ao aprender a lidar com os problemas de adaptação externa e integração interna e que funcionou bem o suficiente para serem considerados válidos e ensinados a novos membros como a forma correta de perceber, pensar e sentir com relação a esses problemas (SCHEIN, 1992, p.2).

Entre os pesquisadores do tema parece prevalecer a noção de que a cultura organizacional é construída por meio da interação entre empregados e dirigentes da organização que compartilham alguns aspectos (chamados de pressupostos, crenças, valores, evidências) capazes de conferir certa identidade à organização. Conceitos que seguem esta linha são tidos como funcionalistas, pois se entende a cultura como uma variável, algo que a organização tem, ao invés de vê-la como uma metáfora raiz, ou seja, algo que a organização é (SMIRCICH, 1983). A cultura organizacional é percebida pelas pessoas através das caraterísticas apresentadas no ambiente da organização e essas características se amplificam de acordo com o crescimento das empresas multinacionais (brasileiras, ou não) e se expressam nas atividades de negócios que envolvem as diferentes culturas (em sentido amplo, entre países, empresas, regiões, por exemplo) (LACERDA, 2011).

Para fins deste estudo, a cultura organizacional pode ser entendida como o conjunto constituído pelo padrão de crenças e valores refletido nas ações, tradições, costumes, 
expectativas e hábitos compartilhados que dão a um grupo particular de pessoas significado e Ihes provê regras de comportamento e orientações apliccáveis a todos os indivíduos que nela interagem. Assim, uma empresa pode ser vista como um grupo integrado que acredita e desenvolve o trabalho conjunto.

\section{Perfil da Amostra}

Os resultados demonstram que as pesquisas sobre cultura organizacional são pouco representativas, representando apenas $3,79 \%$ do total de artigos produzidos e disponibilizados no Portal SPEEL, no período entre 2008 e 2011. A tabela 4 apresenta o total de artigos relacionados à cultura organizacional, assim como, a proporção entre eles, por ano, no referido portal onde foram pesquisados. Cabe salientar que no ano de 2008 o número de artigos foi menor, pois foi a partir deste ano que a produção científica começou a ser concentrada no Portal SPELL.

Tabela 4: Distribuição dos Artigos Pesquisados.

\begin{tabular}{|c|c|c|c|c|}
\hline $\begin{array}{c}\text { LOCAL DE } \\
\text { PESQUISA }\end{array}$ & $\begin{array}{c}\text { PERIOODO } \\
\text { (ANO) }\end{array}$ & $\begin{array}{c}\text { TOTAL DE } \\
\text { ARTIGOS }\end{array}$ & $\begin{array}{c}\text { ARTIGOS } \\
\text { SELECIONADOS }\end{array}$ & $\begin{array}{c}\text { REPRESENTATIVIDADE NO PORTAL } \\
\text { SPELL POR PERÍODO (\%) }\end{array}$ \\
\hline Portal SPELL & 2008 & 1244 & 4 & $0,32 \%$ \\
\hline Portal SPELL & 2009 & 1240 & 13 & $1,05 \%$ \\
\hline Portal SPELL & 2010 & 1291 & 18 & $1,39 \%$ \\
\hline Portal SPELL & 2011 & 1459 & 15 & $1,03 \%$ \\
\hline
\end{tabular}

\section{Autores dos Artigos Analisados}

Entre os artigos analisados, 5 autores destacam-se por apresentar uma produtividade maior, correspondendo a $20 \%$ do total dos artigos selecionados. Entre os autores caracterizados, destacam-se com a quantia de 2 artigos cada: Leilianne Michelle Trindade da Silva (Universidade de São Paulo - USP); Lindomar Pinto da Silva (Universidade Federal da Bahia - UFBA); Luciana Resende Ávila (Universidade Federal de Uberlândia - UFU); Neusa Rolita Cavedon (Universidade Federal do Rio Grande do Sul - UFRGS); Vanderlei dos Santos (Fundação Universidade Regional de Blumenau - FURB).

\section{Assuntos mais Frequentes Relacionados ao Tema}

Quanto aos assuntos relacionados à cultura organizacional, podemos destacar que a maioria dos estudos trata de temas tradicionais sobre a cultura organizacional, sendo o tema cultura nacional, mudança organizacional e aprendizagem organizacional apareceram com maior frequência entre os artigos, representando $30 \%$ da diversidade dos assuntos. Segundo os estudos apresentados a cultura nacional interfere fortemente na cultura de uma organização. Já a mudança organizacional apresenta-se como um assunto atual, uma vez que o contexto ambiental das organizações está tornando-se cada vez mais instável e, por isso, a mudança ocorre com maior frequência. Por fim, a aprendizagem organizacional apresenta-se como uma ferramenta 
cada vez mais importante para as organizações enfrentarem a mutabilidade organizacional e se sobressaírem no mercado. No Gráfico 2 são apresentados os assuntos mais frequentes relacionados ao tema principal.

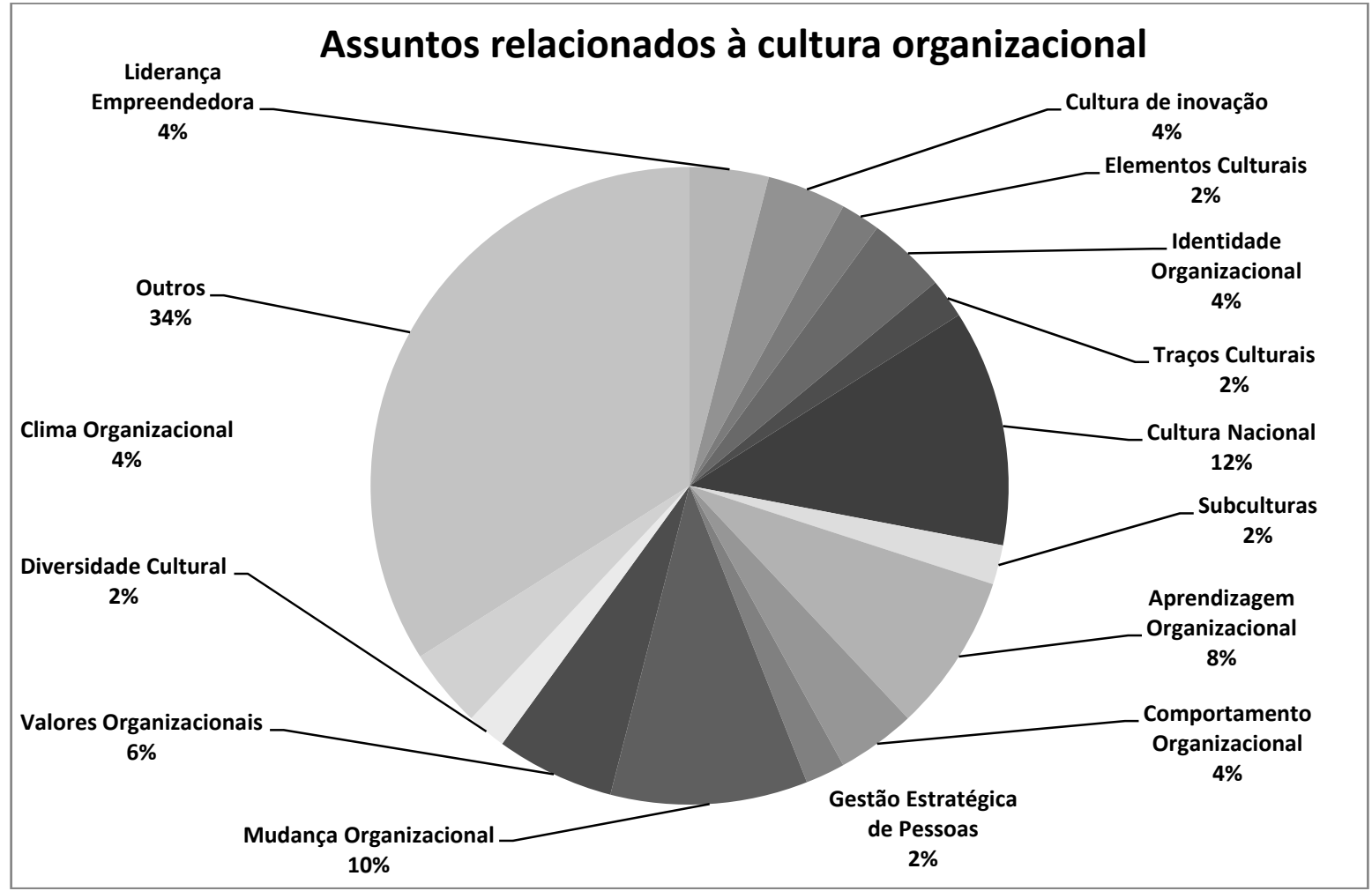

Gráfico 2: Distribuição dos Assuntos Relacionados à Cultura Organizacional.

\section{Distribuição de Artigos por Periódicos}

A partir do levantamento realizado, podemos constatar que alguns periódicos apresentam um volume maior de publicações sobre cultura organizacional. Entre os 26 periódicos analisados, 6 deles concentram $48 \%$ da produção, ou seja, quase metade dos artigos analisados. Ao verificar a distribuição do universo das publicações sobre cultura organizacional observa-se que a Revista Eletrônica de Ciência Administrativa e a Revista de Administração Pública representam cada, 10\% do total de artigos analisados, enquanto a Revista de Administração da UNIMEP e a Revista de Administração Mackenzie representam cada, $8 \%$ do total de artigos e por fim, a Revista de Administração da UFSM e a Revista Alcance representam 6\% cada. A distribuição das publicações sobre cultura organizacional por periódico (Tabela 5) revela diferenças entre os veículos de divulgação quanto à presença de estudos sobre cultura organizacional. Tais diferenças podem ser atribuídas a diversos fatores, entre os quais, a preferência dos autores por determinados periódicos, a especificidade temática de alguns destes periódicos assim como a periodicidade dos mesmos. A Revista de Administração Pública, por exemplo, é uma publicação de caráter bimestral e que pode ter contribuído para que, ela tenha sido uma das que apresentaram maior volume de produção. 
Tabela 5: Distribuição de Artigos por Periódicos.

\begin{tabular}{|l|l|l|}
\hline PERIÓDICOS & $\mathbf{f}(\mathrm{TOTAL})$ & $\mathbf{f}(\%)$ \\
\hline Revista Eletrônica de Ciência Administrativa & 5 & $10 \%$ \\
\hline Revista de Administração Pública & 5 & $10 \%$ \\
\hline Revista de Administração da UNIMEP & 4 & $8 \%$ \\
\hline Revista de Administração Mackenzie & 4 & $8 \%$ \\
\hline Revista de Administração da UFSM & 3 & $6 \%$ \\
\hline Revista Alcance & 3 & $6 \%$ \\
\hline Outros & 26 & $52 \%$ \\
\hline
\end{tabular}

\section{CONCLUSÕES}

O estudo realizado oferece importantes contribuições para uma maior caracterização da produção científica nacional sobre cultura organizacional. Percebe-se que, pelos dados e pela análise feita, existe um número baixo de publicações em periódicos brasileiros sobre cultura organizacional. Os resultados obtidos evidenciam uma diversidade temática e metodológica da produção científica sobre cultura organizacional, bem como a preocupação dos autores em abordar temas atuais como a aprendizagem e mudança organizacional, e temas específicos como a realidade das organizações do Brasil e o estudo da influência da cultura nacional sobre a cultura organizacional. Esta realidade demonstra que os trabalhos sobre cultura organizacional não estão voltados apenas para a reprodução de assuntos, mas estão considerando a diversidade de assuntos relacionados ao tema.

Outro ponto importante constatado, é que o levantamento indica que a produção de conhecimento está voltada tanto para a execução de intervenções, como para impulsionar o desenvolvimento teórico sobre o tema. Isto ficou evidente ao observar que os estudos estavam voltados tanto para o estudo das organizações como para de seus recursos humanos (funcionários). Seria enriquecedor aos resultados se para o desenvolvimento do estudo se contemplassem períodos mais longos, no intuito de identificar tendências e mudanças temáticas da produção científica sobre cultura organizacional. Outro aspecto que poderia contribuir para esta análise seria a inclusão de dissertações e teses sobre o tema, por constituírem importantes fontes de produção científica.

\section{REFERÊNCIAS}

ARAÚJO, C. A.. Bibliometria: evolução história e questões atuais. Revista em Questão, Porto Alegre, v.12, n.1, p.11-32, 2006.

BELTRAO, D. C.; CARDOSO, A.. Análise da existência de subculturas em uma multinacional europeia sediada no Brasil. Organizações em contexto. São Paulo, v.5, n.9, p.61-91, 2009.

FERREIRA, A.G. C.. Bibliometria na avaliação de periódicos científicos. Revista de Ciência da Informação, v.11, n.3, 2010.

FIGUEIREDO, N. M.. Desenvolvimento e Avaliação de Coleções. Rio de Janeiro: Rabiskus, 1993. 
GNECCO JÚNIOR.. Análise bibliométrica da produção científica nos Colóquios I .a IX In: COLÓQUIO INTERNACIONAL SOBRE GESTÃO. 5. Anais. Florianópolis: Universidade Federal de Santa Catarina, 2010.

LACERDA, D. P.. Cultura organizacional: sinergias e alergias entre Hofstede e Trompenaars. Revista de Administração Pública. Rio de Janeiro v.45, n.5, p.1285-1301, 2011.

LAPLANTINE, F.. Aprender Antropologia. São Paulo: Brasiliense, 1995.

MACIEL, L. R.; MACIEL, M. R.; FROEMMING, L. M. S.. Valor de Marca e Cultura Organizacional como Baluartes Estratégicos no Ciclo de Vida de uma Empresa: os 92 anos da Indústria de Balas Soberana.

Revista Eletrônica de Ciência Administrativa. Paraná, v.10, n.1, p.128-139, 2011.

MORGAN, G.. Imagens da Organização. São Paulo: Atlas, 1996.

ROBBINS, S. P.. O Processo Administrativo. São Paulo: Atlas, 1990.

SILVA L. P.; FADUL, E.. A Produção Científica Sobre Cultura Organizacional em Organizações Públicas no Período de 1997 a 2007: um Convite à Reflexão. Revista de Administração Contemporânea. Curitiba, v. 14, n.5, p.651-669, 2010.

SMIRCICH, L.. Concepts of culture and organizational analysis. Administrative Science Quarterly, v.28, n.3, p.339-359, 1983.

VERGARA, S. C.. Projetos e relatórios de pesquisa em administração. 4 ed. São Paulo: Atlas, 2003. 